\title{
Streamlining your business for success
}

\author{
Mark Pykett \& Derek Lee \\ In the current fund-raising environment, focusing on rapid decision making, spreading risk across several assets and \\ introducing innovative trial designs could pay dividends for biotech entrepreneurs.
}

$T_{p}^{\mathrm{h}}$ he financing environment for the biopharmaceutical sector has changed dramatically over the past decade. Though some assert that unprecedented amounts of capital are sitting on the sidelines, recruiting that capital into companies has proven difficult. Furthermore, although investors will still invest in what they believe to be unique opportunities, there is also tremendous pressure overall to do more with less.

Thus, if you are an entrepreneur hoping to succeed in the present fund-raising environment, you must emphasize improvements in productivity and efficiency. In this article, we highlight several business practices, some widely used in other industries, that can help reshape your odds for success.

\section{Both win and fail fast}

Biotech is legendary for its ability to persist under a generally losing proposition. A familiar path goes like this: company raises money around a new product or technology and its intellectual property; company develops lead product to proof of principle in humans; lead product fails in clinical trial; company business model is revised and new funding raised; a new (and often similar) trial is conducted; the trial again fails; company in-licenses a different product or technology; the process recommences from the beginning.

Because biotech pushes the envelope of science and technology, we tolerate a high degree of failure. But not being smart about how we fail is no longer acceptable. Companies need to focus on ways to efficiently reach key milestones-those decision points that represent

Mark Pykett is CEO and Derek Lee is chief financial and corporate development officer at Talaris Advisors, Hopkinton, Massachusetts, USA. e-mail:mpykett@talarisadvisors.comor dlee@talarisadvisors.com the proverbial 'go or no-go' forks in the road. This is especially true during the so-called valley of death, or the span of time between discovery stage and attainment of clinical proof of concept.

From the outset, you need to ask the right questions while developing drugs toward proof of concept. To optimize efficiency, you need to emphasize investigating those questions that lead to quick success or rapid failure. In some ways, this is the heart of the 'kill shot theory': What is the key question that will, if answered in the negative, cause you to shut down the program, or, if answered positively, provide the required support to make additional investment?

There is no uniform kill shot across drug development programs, so you'll need to develop rigorous criteria explicitly defining success or failure. We once worked on developing a recombinant protein for antiangiogenic indications. The protein had been engineered from a biochemistry standpoint for optimal stability and function in cell-based assays, and it had been tested in several animal models for safety. But it had never been tested in definitive efficacy animal models. The key question to demonstrate preclinical efficacy had not been asked, and it was not clear what was being developed. In this case, we asked a hard kill shot question: Does the protein have an effect on angiogenesis and tumor growth in the gold standard animal model? When results did not meet the criteria for success, the program was shut down. Had that key question been asked earlier, time and money surely would have been saved.

It's not just about wasted investment; it's also about opportunity cost. No organization has unlimited resources to pursue all potential projects (this is especially true for small firms), so when a project is developed, others must be passed up. Each day and dollar spent on a given program is effectively an opportunity cost.
Another way to improve efficiency is to spend less time and money by implementing strategies that get to the key clinical trial decision points quicker. This can be done by using statistical methodologies to enroll fewer trial subjects. For example, we have actively used adaptive designs backed by Bayesian statistics (often used in device development). Adaptive designs can expediently decrease both samples sizes and overall enrollment times, which is particularly attractive in trials for slow-enrolling indications. We developed a trial for a biologic drug in an orphan indication for neurotrauma by using Bayesian statistics to design an adaptive dose-selection study that cut an estimated two years off the development timeline (Box 1).

In our experience, statistical leverage can be used to accelerate milestones, reduce costs and shrink sample sizes. In one case involving the development of a diagnostic agent, a standard registration trial was designed around conventional outcome measures of sensitivity and specificity. The sample size was expected to be more than 2,400 subjects, and the timeline suggested more than 8 years. We designed a trial based on area-under-the-curve end points to cut cost and the timeline (Box 2).

Even so, it is quite likely the expertise required to complete such statistical analyses will lie outside your organization, so you'll need to engage advisors or consultants experienced in advanced statistical designs, especially those who have previously assisted clinical drug development studies.

Often, the reason hard decisions regarding resource allocation aren't made is because the company is a one-trick pony, and its success (and the careers of its managers) are tied to a binary outcome. In such cases, managers are often unwilling or incapable of being objective. If the program fails, after all, their careers are in jeopardy. This inherent misalignment of management interests with those of investors can 


\section{Box 1 Cutting cost in neurotrauma}

Neurotrauma is fraught with complexities in indications ranging from stroke to traumatic brain injury to spinal cord injury. The cost of conducting clinical trials is extensive, particularly with traditional approaches, so when working in a neurotrauma program, we looked for leverage in existing data by completing an interim analysis to identify informative efficacy signals in certain subsets of patients with better outcomes.

This had key implications for the design, sample size and cost of further clinical work, and it became the focus of the planning for the next-stage clinical study. We employed Bayesian statistics - which allow inferences and decisions to be based on observations or experiments and also prior knowledge or expertise - to drive an adaptive design trial. This effort culminated in an integrated phase $2 / 3$ trial design intended to serve as the basis for dose selection and marketing approval. This resulted in a decrease in the sample size, trial duration and cost by $80 \%$ compared with those of standard parallel group clinical trial designs. Working with regulatory authorities, we then established the clinical end points and dose-selection strategy for the phase 2 trial and the basis for a single phase 3 trial and marketing approval.

be addressed by employing a strategy found in many other businesses, including the financial industry, and it deals with odds.

\section{Stacking the odds}

We don't suggest that biotech drug development equates to gambling (although others might), but the principles of odds do apply. It's true that in gambling there are ways to shift the odds, such as by playing blackjack or poker, in which historical cards dealt and your ability to read the table can slant things (however slightly) in your favor. Conversely, as exhilarating as the prospect of going all in for a hand may be, it is most often a sure way to lose everything.

In the financial investing industry, risks are mitigated by spreading money across assets so that no single project drives the failure of the invested funds. But biotech managers often do not address risk mitigation by spreading investment across multiple programs. Yet it is clear that probabilities apply to biotech product development as much as to finances. Venture capitalists investing in the biotech sector balance their risk by plac- ing money across companies. You never hear of a venture group investing its entire fund in a single company, no matter how bullish they may be on the opportunity.

Assume, for example, that the chances of success for a series of clinical development programs in a company boil down to one in four assets, in isolation and without regard for intrinsic technology attributes (we recognize this is an optimistically favorable success rate, but indulge us). In this approach, each portfolio of four or more assets will have at least one that succeeds, meaning that even in a worst-case scenario there will be downside protection for investors (and for the CEO).

This type of resource deployment, by the way, is also a more efficient use of current capital. In a company running just one development project, a significant amount of money is used simply to keep the team in place, even during periods when they are not fully in use. Imagine a business paying its people $100 \%$ of their salary for a $60 \%$ level of effort. Maybe certain businesses can get away with this, but it ultimately diminishes returns.

\section{Box 2 Save time, patients and money}

A promising agent being developed to address diagnosing several high-prevalence neurological disorders had been through numerous clinical studies without reaching the standards for approval, and thus the programs were stalled. The sponsor estimated that registration of the agent using conventional development plans would require upward of 2,400 subjects and $\$ 120$ million over 8 years. Under those conditions, the program had a negative net present value. We conducted a comprehensive audit to identify avenues to improve efficiency and returns and then worked closely with field experts to develop an innovative statistical strategy that used area-under-the-curve outcome measures to demonstrate agent efficacy as the basis for approval.

After substantial work with federal regulators, we reached a consensus on a special protocol assessment that agreed on the protocol design and statistical analysis plan. The overall design reduced the number of subjects to 400 and the cost to about $\$ 20$ million. These changes decreased the investment risk and defined an integrated development path.

By pursuing several development programs at once, you can exploit the cyclical nature of projects and keep your team's workload at or near full capacity. What happens when two or more projects each require the team's full attention? We argue this is rare, but effective management and the use of outsourced help can guide you through the crunch.

Companies also spend money supporting infrastructure and team building-indirect expenditures that usually don't generate returns on assets on par with direct expenditures. Pursuing multiple programs means the team is working at closer to full capacity, and thus a lower proportion of funding is devoted to carrying costs-another reason for avoiding the one-trick pony approach.

This is all fine and well in theory, you may counter, but as an entrepreneur at a startup you do not have three or four distinct assets or technologies on hand. Although you may not have a rounded portfolio, you may actually have a basket of potential applications you can go after. It's possible to apply the same principles of risk mitigation and probability distribution to a single technology by deploying it in multiple development programs with different risk profiles. For example, we recently worked with a company developing a technology for growing stem cells that could be applied to a range of applications, some of which were incremental improvements over the state of the art (low risk) and some that would be potential breakthroughs (high risk). The low-risk applications had smaller market potential and lower potential returns but provided downside protection against the failure of the more farreaching applications. This gave investors the multiple 'shots on goal' they sought as well as a safety net that improved the risk profile of the overall investment.

In evaluating how to position your applications and development plans, take time to think about how high-risk/high-return applications might be balanced with low-risk/ low-return programs, especially if the latter assets cost less to develop. This exercise will cause you to look carefully at how you are balancing risk and allocating your resources, and it might go a long way toward winning the investment you need and increasing your probability for success.

\section{Non-integration equals disintegration}

Not all points of leverage need be as strategic as those described above; it can be very challenging to assemble baskets of quality assets, and some programs in fact are better served by marching through conventional development processes, especially in areas that have strong precedents. 
Regardless, in today's environment, you'll most likely need to outsource development activities as well as minimize internal infrastructure. As noted earlier, infrastructure often does not return inherent value to the underlying asset or to investors, and recruiting internal teams that are busy only $50 \%$ of the time multiplies carrying costs. Outsourcing allows companies to keep costs variable.

Even so, effective outsourcing is difficult to achieve. There are a growing number of expert, capable service providers, and contract research organizations can generate products of excellent quality, but parsing out areas of development to different contractors has its own risks. For example, if the nonclinical product of contractor $\mathrm{A}$ is not aligned with the clinical product of contractor B, it's likely they will not be integrated and aspects of your project may need to be repeated. The result may actually be a loss of efficiency. You'll need a keen eye and strong hand to ensure that all aspects of the project achieve the required modicum of integration.

Another tactic to improve returns (and keep your biotech healthy) is to carefully calibrate the timing and magnitude of your project activities. Under a typical drug development model, there is a sequence to traditional development steps that often involves up-front investments in downstream activities. For example, it's usually common practice to invest in manufacturing activities that will far exceed the requirements of getting to the next milestone. This is virtuous in its optimism toward success, but the truth is that most drug development programs fail. As such, investing early in activities that are required only when a drug passes all its tests can represent an over-allocation of resources.

Here's an example. We've run across drug development programs in which the company manufactures a clinical drug supply before entering phase 1 trials that is sufficient to carry the program through pivotal registration studies. This can be rationalized because if the program is successful, the company doesn't want to wait for new lots to be made, and besides, multiple manufacturing runs will be required for approval. But what happens when the program doesn't move forward? These resources are useless and wasted; given the probabilities, this happens more often than not.

The same can be true of nonclinical programs. The industry has evolved to require a certain standard of nonclinical testing both before human clinical studies and for drug registration, though not all programs have the same standards. Some programs can progress quickly to clinical proof of concept before completing the full battery of nonclinical tests. For example, in programs we have built, we've pursued exploratory investigational new drug applications using limited nonclinical data, which allowed us to generate human clinical results that validated the drug's merit instead of funding more extensive nonclinical testing that ordinarily would have preceded human studies.

To discuss the contents of this article, join the Bioentrepreneur forum on Nature Network: http://network.nature.com/groups/bioentrepreneur/forum/topics
Although we're not alone in our thinking (Eli Lilly's Chorus group, for example, has used this approach, as have smaller organizations from contract research outfits like Cato Research to small, independent biotechs), this strategy is not the norm.

We view this as effective risk management: if the drug fails to get through the well-defined, pre-specified kill shot, the program is terminated and money that would have been spent on downstream activities is saved. If the program passes a key test, you must then go back and do certain activities you have deferred, but this need not necessarily extend the project timeline. Given the prevailing low odds of success, in the long run this trade-off in time is worth the savings achieved. By using such hedges across time, you'll know sooner if you need to spend that money.

\section{Conclusions}

As an entrepreneur, you are far likelier to succeed if you change the odds by making investments based on probabilities instead of emotions. The focus should be less on shiny infrastructure and more on virtualization and taking programs forward in the shortest time possible. By making hard decisions and killing projects sooner (and at lower cost), you can devote more resources to assets that generate successes for both you and your investors. 10. 